\title{
Lyapunov-type inequalities for quasilinear elliptic equations with Robin boundary condition
}

\author{
Ülkü Dinlemez Kantar ${ }^{1 *}$ and Tülay Özden²
}

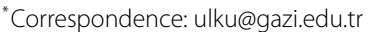 'Department of Mathematics, Faculty of Sciences, Gazi University, 06500 Teknikokullar, Ankara, Turkey Full list of author information is available at the end of the article}

\begin{abstract}
The aim of this study is to prove Lyapunov-type inequalities for a quasilinear elliptic equation in $\mathbb{R}^{2}$. Also the lower bound for the first positive eigenvalue of the boundary value problem is obtained.
\end{abstract}

MSC: Primary 35J40; 35J66; secondary 46E35

Keywords: Lyapunov-type inequalities; elliptic equations; Robin boundary conditions

\section{Introduction}

In [1], Lyapunov proved that, if $p(x)$ is a nonnegative and continuous function and $u(x) \in$ $C(I, \mathbb{R})$, a necessary condition for the following boundary value problem:

$$
\left\{\begin{array}{l}
u^{\prime \prime}(x)+p(x) u(x)=0, \quad u(x) \neq 0, \forall x \in I \\
u\left(a_{1}\right)=0=u\left(b_{1}\right)
\end{array}\right.
$$

to have nontrivial solutions is

$$
4 /\left(b_{1}-a_{1}\right) \leq \int_{a_{1}}^{b_{1}} p(x) \mathrm{d} x
$$

where $I=\left[a_{1}, b_{1}\right]$.

Since Lyapunov's study, because the inequality of (1.2) plays a key role for the qualitative properties, such as oscillatory and disconjugacy etc., of differential equations' solutions, several authors focused on the inequality of (1.2). Those authors improved and generalized the inequality of (1.2) in $\mathbb{R}$. In this work the literature of the one-dimensional case is not studied in detail but it is listed in the references for the interested reader. See [1-17] and the references cited therein.

In addition to studies in $\mathbb{R}$, several authors [18-24] have extended the inequality of (1.2) in $\mathbb{R}^{n}$ recently. To the best of our knowledge, it was extended by Cañada, Montero, and Villegas [19] for the first time. In [19] Cañada et al. considered the linear elliptic problem

(c) The Author(s) 2017. This article is distributed under the terms of the Creative Commons Attribution 4.0 International License (http://creativecommons.org/licenses/by/4.0/), which permits unrestricted use, distribution, and reproduction in any medium, provided you give appropriate credit to the original author(s) and the source, provide a link to the Creative Commons license, and indicate if changes were made. 
as follows:

$$
\left\{\begin{array}{l}
-\triangle u=a(x) u, \quad x \in \Omega \\
\partial u / \partial n=0, \quad x \in \partial \Omega
\end{array}\right.
$$

where $\Omega \subset \mathbb{R}^{N}$ is a smooth bounded domain with $N \geq 2$ and the function $a: \Omega \rightarrow \mathbb{R}$ belongs to the set

$$
\Lambda=\left\{a \in L^{N / 2}(\Omega) \backslash\{0\}: \int_{\Omega} a(x) \mathrm{d} x \geq 0 \text { and (1.3) has a nontrivial solution }\right\}
$$

if $N \geq 3$,

$$
\begin{aligned}
\Lambda= & \left\{a: \Omega \rightarrow \mathbb{R} \text { s.t. } \exists q \in(1, \infty] \text { with } a \in L^{q}(\Omega) \backslash\{0\}: \int_{\Omega} a(x) \mathrm{d} x \geq 0\right. \\
& \text { and (1.3) has a nontrivial solution }\}
\end{aligned}
$$

if $N=2$, we define

$$
\beta_{q}:=\inf _{a \in \wedge \cap L^{q}(\Omega)}\|a\|_{L^{q}(\Omega)}, \quad 1 \leq q \leq \infty
$$

Their main result is as follows.

\section{Theorem A The following statements hold.}

(1) If $N=2$ then $\beta_{q}>0 \Leftrightarrow 1<q \leq \infty$. If $N \geq 3$ then $\beta_{q}>0 \Leftrightarrow \frac{N}{2} \leq q \leq \infty$.

(2) If $\frac{N}{2}<q \leq \infty$ then $\beta_{q}$ is attained. In this case, any function $a \in \wedge \cap L^{q}(\Omega)$ from which $\beta_{q}$ is attained has one of the following forms:

(i) $a(x)=\lambda_{1}$, if $p=\infty$, where $\lambda_{1}$ is the first strictly positive eigenvalue of (1.3).

(ii) $a(x)=|u(x)|^{\frac{2}{p-1}}$, if $\frac{N}{2}<q<\infty$, where $u$ is a solution of the problem as follows:

$$
\left\{\begin{array}{l}
-\Delta u=|u(x)|^{2 /(p-1)} u, \quad x \in \Omega \\
\partial u / \partial n=0, \quad x \in \partial \Omega
\end{array}\right.
$$

(3) The map $\left(\frac{N}{2}, \infty\right) \rightarrow \mathbb{R}, p \mapsto \beta_{p}$, is continuous and the map $\left[\frac{N}{2}, \infty\right) \rightarrow \mathbb{R}$, $p \mapsto|\Omega|^{\frac{-1}{p}} \beta_{p}$, is strictly increasing.

(4) The limits $\lim _{p \rightarrow \infty} \beta_{p}$ and $\lim _{p \rightarrow\left(\frac{N}{2}\right)^{+}} \beta_{p}$ always exist and take the values

(i) $\lim _{p \rightarrow \infty} \beta_{p}=\beta_{\infty}$, if $N \geq 2$,

(ii) $\lim _{p \rightarrow\left(\frac{N}{2}\right)^{+}} \beta_{p} \geq \beta_{\frac{N}{2}}>0$, if $N \geq 3, \lim _{p \rightarrow 1^{+}} \beta_{p}=0$, if $N=2$.

Here, we also note that in the study Cañada et al. they proved that the relation between the $p$ and $\frac{N}{2}$ plays a crucial role. They also considered the equation in (1.3) with zero Dirichlet boundary condition. They presented similar inequalities at their study. Then others established Lyapunov-type inequalities for different equations with boundary conditions. For more information about the studies in $\mathbb{R}^{n}$, the interested reader can refer to [18-24] and the references cited therein. 
The aim of this paper is to prove a Lyapunov-type inequality for the two-dimensional quasilinear elliptic problem as follows:

$$
\left\{\begin{array}{l}
-\left(\Phi_{p}\left(u_{x y}\right)\right)_{y}=r(x, y) \Phi_{p}(u), \quad(x, y) \in \Omega \\
u\left(a_{1}, y\right)=0=u\left(b_{1}, y\right), \quad a_{2} \leq y \leq b_{2} \\
u_{x}\left(x, a_{2}\right)=0=u_{x}\left(x, b_{2}\right), \quad a_{1} \leq x \leq b_{1}
\end{array}\right.
$$

where $\Omega=\left[a_{1}, b_{1}\right] \times\left[a_{2}, b_{2}\right]$ and $r(x, y)$ is a measurable function on $\Omega$, and $\Phi_{p}(u(x, y))=$ $|u(x, y)|^{p-2} u(x, y)$ for $p>1$. In addition to this, we note that by a solution of the problem (1.8), we mean that $u(x, y) \in W^{3, p}(\Omega)$ in that

$$
W^{3, p}(\Omega)=\left\{u: u, u_{x}, u_{x y} \text { and } u_{x y y} \in L^{p}(\Omega)\right\}
$$

As usual, $L^{p}(\Omega)$ is a space of Lebesgue measurable functions.

\section{Main results}

Now, we give a key lemma as a proof of our main conclusions.

Lemma 1 Assume that $u(x, y) \in W^{3, p}(\Omega)$, it satisfies the boundary conditions in (1.8) and $u(x, y) \neq 0$ for $\forall(x, y) \in \Omega^{0}$. Then

$$
\begin{aligned}
& (4|u(x, y)|)^{p} /\left(b_{1}-a_{1}\right)^{p-1}\left(b_{2}-a_{2}\right)^{p-1} \leq \int_{a_{1}}^{b_{1}} \int_{a_{2}}^{b_{2}}\left|u_{x y}\right|^{p} \mathrm{~d} y \mathrm{~d} x, \\
& \left(2^{p} /\left(b_{2}-a_{2}\right)^{p-1}\right) \int_{a_{1}}^{b_{1}}\left|u_{x}\right|^{p} \mathrm{~d} x \leq \int_{a_{1}}^{b_{1}} \int_{a_{2}}^{b_{2}}\left|u_{x y}\right|^{p} \mathrm{~d} y \mathrm{~d} x,
\end{aligned}
$$

hold, respectively, where $\Omega^{0}$ is the set of all interior points of $\Omega$.

Proof Let $(x, y) \in \Omega$. Since $u(x, y)$ satisfies the boundary conditions in (1.8), it is easy to see

$$
u(x, y)=\int_{a_{1}}^{x} \int_{a_{2}}^{y} u_{t s} \mathrm{~d} s \mathrm{~d} t
$$

taking the absolute value, we obtain

$$
|u(x, y)| \leq \int_{a_{1}}^{x} \int_{a_{2}}^{y}\left|u_{t s}\right| \mathrm{d} s \mathrm{~d} t
$$

Similarly, we get

$$
\begin{aligned}
& |u(x, y)| \leq \int_{a_{1}}^{x} \int_{y}^{b_{2}}\left|u_{t s}\right| \mathrm{d} s \mathrm{~d} t, \\
& |u(x, y)| \leq \int_{x}^{b_{1}} \int_{a_{2}}^{y}\left|u_{t s}\right| \mathrm{d} s \mathrm{~d} t,
\end{aligned}
$$

and

$$
|u(x, y)| \leq \int_{x}^{b_{1}} \int_{y}^{b_{2}}\left|u_{t s}\right| \mathrm{d} s \mathrm{~d} t .
$$


Adding (2.3)-(2.6), we have

$$
4|u(x, y)| \leq \int_{a_{1}}^{b_{1}} \int_{a_{2}}^{b_{2}}\left|u_{x y}\right| \mathrm{d} y \mathrm{~d} x
$$

Then, applying Hölder's inequality

$$
\int_{a_{1}}^{b_{1}}|f(t) g(t)| \mathrm{d} t \leq\left(\int_{a_{1}}^{b_{1}}|f(t)|^{q} \mathrm{~d} t\right)^{1 / q}\left(\int_{a_{1}}^{b_{1}}|g(t)|^{p} \mathrm{~d} t\right)^{1 / p}
$$

to the right-hand side of (2.7), we get

$$
(4|u(x, y)|)^{p} \leq\left(b_{1}-a_{1}\right)^{p-1} \int_{a_{1}}^{b_{1}}\left[\int_{a_{2}}^{b_{2}}\left|u_{x y}\right| \mathrm{d} y\right]^{p} \mathrm{~d} x .
$$

Applying Hölder's inequality to the right hand side of (2.9) again, we obtain

$$
\frac{(4|u(x, y)|)^{p}}{\left(b_{1}-a_{1}\right)^{p-1}\left(b_{2}-a_{2}\right)^{p-1}} \leq \int_{a_{1}}^{b_{1}} \int_{a_{2}}^{b_{2}}\left|u_{x y}\right|^{p} \mathrm{~d} y \mathrm{~d} x .
$$

Thereby, the proof of (2.1) is completed.

Similarly we have

$$
\left|u_{x}(x, y)\right| \leq \int_{a_{2}}^{y}\left|u_{x s}\right| \mathrm{d} s
$$

and

$$
\left|u_{x}(x, y)\right| \leq \int_{y}^{b_{2}}\left|u_{x s}\right| \mathrm{d} s
$$

Adding (2.10) and (2.11), we get

$$
2\left|u_{x}(x, y)\right| \leq \int_{a_{2}}^{b_{2}}\left|u_{x s}\right| \mathrm{d} s
$$

Applying Hölder's inequality to the right hand side of (2.12) and integrating from $a_{1}$ to $b_{1}$, we have

$$
\frac{2^{p}}{\left(b_{2}-a_{2}\right)^{p-1}} \int_{a_{1}}^{b_{1}}\left|u_{x}\right|^{p} \mathrm{~d} x \leq \int_{a_{1}}^{b_{1}} \int_{a_{2}}^{b_{2}}\left|u_{x y}\right|^{p} \mathrm{~d} y \mathrm{~d} x .
$$

Consequently, the proof of (2.2) is completed.

Theorem 1 If $u(x, y) \in W^{3, p}(\Omega)$ is a nontrivial solution of the problem (1.8), then the following inequality:

$$
2^{2 p+1} /\left(b_{1}-a_{1}\right)^{p-1}\left(b_{2}-a_{2}\right)^{p} \leq \int_{a_{1}}^{b_{1}} \int_{a_{2}}^{b_{2}}|r(x, y)|^{q} \mathrm{~d} y \mathrm{~d} x
$$

holds, where $q$ is the Hölder conjugate of $p$. 
Proof Let $u(x, y) \in W^{3, p}(\Omega)$ is a nontrivial solution of the problem (1.8). Multiplying the equation in (1.8) by $u_{x}$ and integrating on $\Omega$,we obtain

$$
\int_{a_{1}}^{b_{1}} \int_{a_{2}}^{b_{2}}-\left(\left|u_{x y}\right|^{p-2} u_{x y}\right)_{y} u_{x} \mathrm{~d} y \mathrm{~d} x=\int_{a_{1}}^{b_{1}} \int_{a_{2}}^{b_{2}} r(x, y)|u|^{p-2} u u_{x} \mathrm{~d} y \mathrm{~d} x
$$

Then, applying partial integration in $\int_{a_{2}}^{b_{2}}-\left(\left|u_{x y}\right|^{p-2} u_{x y}\right)_{y} u_{x} \mathrm{~d} y$ and using the boundary conditions in (1.8), we have

$$
\int_{a_{1}}^{b_{1}} \int_{a_{2}}^{b_{2}}\left|u_{x y}\right|^{p} \mathrm{~d} y \mathrm{~d} x=\int_{a_{1}}^{b_{1}} \int_{a_{2}}^{b_{2}} r(x, y)|u|^{p-2} u u_{x} \mathrm{~d} y \mathrm{~d} x
$$

By taking the absolute value on right hand side of (2.15), we get

$$
\int_{a_{1}}^{b_{1}} \int_{a_{2}}^{b_{2}}\left|u_{x y}\right|^{p} \mathrm{~d} y \mathrm{~d} x \leq \int_{a_{1}}^{b_{1}} \int_{a_{2}}^{b_{2}}|r(x, y)||u|^{p-1}\left|u_{x}\right| \mathrm{d} y \mathrm{~d} x
$$

Hence, applying Hölder's inequality to the right hand side of (2.16), we find

$$
\begin{aligned}
& \int_{a_{1}}^{b_{1}} \int_{a_{2}}^{b_{2}}\left|u_{x y}\right|^{p} \mathrm{~d} y \mathrm{~d} x \\
& \quad \leq\left(\int_{a_{1}}^{b_{1}} \int_{a_{2}}^{b_{2}}|r(x, y)|^{q}|u|^{(p-1) q} \mathrm{~d} y \mathrm{~d} x\right)^{1 / q}\left(\int_{a_{1}}^{b_{1}} \int_{a_{2}}^{b_{2}}\left|u_{x}\right|^{p} \mathrm{~d} y \mathrm{~d} x\right)^{1 / p} .
\end{aligned}
$$

Now, considering only the second term of right hand side in (2.17) from Fubini's theorem, we can rewrite the inequality (2.17) as follows:

$$
\begin{aligned}
& \int_{a_{1}}^{b_{1}} \int_{a_{2}}^{b_{2}}\left|u_{x y}\right|^{p} \mathrm{~d} y \mathrm{~d} x \\
& \quad \leq\left(\int_{a_{1}}^{b_{1}} \int_{a_{2}}^{b_{2}}|r(x, y)|^{q}|u|^{(p-1) q} \mathrm{~d} y \mathrm{~d} x\right)^{1 / q}\left(\int_{a_{2}}^{b_{2}}\left[\int_{a_{1}}^{b_{1}}\left|u_{x}\right|^{p} \mathrm{~d} x\right] \mathrm{d} y\right)^{1 / p} .
\end{aligned}
$$

Hence, using the inequality (2.2) in (2.18), we obtain

$$
\begin{aligned}
& \left(\int_{a_{1}}^{b_{1}} \int_{a_{2}}^{b_{2}}\left|u_{x y}\right|^{p} \mathrm{~d} y \mathrm{~d} x\right)^{(p-1) / p} \\
& \quad \leq\left(\left(b_{2}-a_{2}\right) / 2\right)\left(\int_{a_{1}}^{b_{1}} \int_{a_{2}}^{b_{2}}|r(x, y)|^{q}|u|^{(p-1) q} \mathrm{~d} y \mathrm{~d} x\right)^{1 / q} .
\end{aligned}
$$

Then, replacing the point of $(x, y)$, which is used in Lemma 1 , with the maximum point of $|u(x, y)|$, from (2.1), we get

$$
\begin{aligned}
& (4 \max |u(x, y)|)^{(p-1)} /\left(b_{1}-a_{1}\right)^{(p-1) / q}\left(b_{2}-a_{2}\right)^{(p-1) / q} \\
& \quad \leq\left(\int_{a_{1}}^{b_{1}} \int_{a_{2}}^{b_{2}}\left|u_{x y}\right|^{p} \mathrm{~d} y \mathrm{~d} x\right)^{(p-1) / p} .
\end{aligned}
$$


Then, using the inequality (2.20) in the inequality (2.19), we have

$$
\begin{aligned}
& (4 \max |u(x, y)|)^{(p-1)} /\left(b_{1}-a_{1}\right)^{(p-1) / q}\left(b_{2}-a_{2}\right)^{(p-1) / q} \\
& \leq\left(\left(b_{2}-a_{2}\right) / 2\right)\left(\int_{a_{1}}^{b_{1}} \int_{a_{2}}^{b_{2}}|r(x, y)|^{q}|u|^{(p-1) q} \mathrm{~d} y \mathrm{~d} x\right)^{1 / q} \\
& \leq\left(\left(b_{2}-a_{2}\right) / 2\right)\left(\int_{a_{1}}^{b_{1}} \int_{a_{2}}^{b_{2}}|r(x, y)|^{q} \mathrm{~d} y \mathrm{~d} x\right)^{1 / q}(\max |u(x, y)|)^{(p-1)} .
\end{aligned}
$$

Since $u(x, y)$ is a nontrivial solution, we have $\max |u(x, y)| \neq 0$. Therefore, we obtain

$$
2^{2 p+1} /\left(b_{1}-a_{1}\right)^{p-1}\left(b_{2}-a_{2}\right)^{p} \leq \int_{a_{1}}^{b_{1}} \int_{a_{2}}^{b_{2}}|r(x, y)|^{q} \mathrm{~d} y \mathrm{~d} x .
$$

Thus, the proof is completed.

Corollary 1 Let $\lambda_{1}$ be the first eigenvalue of the equation that is defined on $\Omega$ as follows:

$$
-\left(\Phi_{p}\left(u_{x y}\right)\right)_{y}=\lambda_{1} r(x, y) \Phi_{p}(u)
$$

where $\Omega$ is a domain, which is defined in the beginning of the paper, and with the boundary conditions in (1.8). Then we have

$$
2^{2 p+1} /\left(b_{1}-a_{1}\right)^{p-1}\left(b_{2}-a_{2}\right)^{p}\|r(x, y)\|_{L^{q}(\Omega)}^{q} \leq \lambda_{1} .
$$

Remark 1 If we take the Dirichlet boundary conditions, which are $u\left(a_{1}, y\right)=0=u\left(b_{1}, y\right)$ and $u\left(x, a_{2}\right)=0=u\left(x, b_{2}\right)$, instead of the Robin boundary conditions in the problem (1.8), then we obtain the identical conclusions given above.

Remark 2 The result, which is obtained in this study, is also the necessary condition for the problem of (1.8) to have a nontrivial solution.

\section{Competing interests}

The authors declare that they have no competing interests.

\section{Authors' contributions}

Both authors contributed equally to the writing of this paper. Both authors read and approved the final manuscript.

\section{Author details}

${ }^{1}$ Department of Mathematics, Faculty of Sciences, Gazi University, 06500 Teknikokullar, Ankara, Turkey. ${ }^{2}$ Department of Mathematics, Institute of Sciences, Gazi University, 06500 Teknikokullar, Ankara, Turkey.

\section{Acknowledgements}

The authors would like to thank the referee for the careful reading of this paper and for the valuable suggestions to improve the presentation and style of the paper.

Received: 11 November 2016 Accepted: 12 February 2017 Published online: 16 February 2017

\section{References}

1. Lyapunov, AM: Problème général de la stabilité du mouvement. Ann. Fac. Sci. Univ. Toulouse 2, 203-407 (1907)

2. Hartman, P, Wintner, A: On an oscillation criterion of Lyapunov. Am. J. Math. 73, 885-890 (1951)

3. Cheng, SS: Lyapunov inequalities for differential and difference equations. Fasc. Math. 23, 25-41 (1991)

4. Kwong, MK: On Lyapunov's inequality for disfocality. J. Math. Anal. Appl. 83, 486-494 (1981)

5. Panigrahi, S: Lyapunov-type integral inequalities for certain higher order differential equations. Electron. J. Differ. Equ. 2009, 28 (2009) 
6. Parhi, N, Panigrahi, S: Disfocality and Liapunov-type inequalities for third-order equations. Appl. Math. Lett. 16 227-233 (2003)

7. Parhi, N, Panigrahi, S: Lyapunov-type inequality for higher order differential equations. Math. Slovaca 52, 31-46 (2002)

8. Parhi, N, Panigrahi, S: Lyapunov-type inequality for delay-differential equations of third order. Czechoslov. Math. J. 52, 385-399 (2002)

9. Parhi, N, Panigrahi, S: On Lyapunov-type inequality for third-order differential equations. J. Math. Anal. Appl. 233, 445-460 (1999)

10. Lee, C, Yeh, C, Hong, C, Agarwal, RP: Lyapunov and Wirtinger inequalities. Appl. Math. Lett. 17, 847-853 (2004)

11. Yang, X, Kim, Y, Lo, K: Lyapunov-type inequalities for a class of quasilinear systems. Math. Comput. Model. 53, $1162-1166(2011)$

12. Yang, X: On inequalities of Lyapunov-type. Appl. Math. Comput. 134, 293-300 (2003)

13. Yang, X: On Liapunov-type inequality for certain higher-order differential equations. Appl. Math. Comput. 134, 307-317 (2003)

14. Pachpatte, BG: On Lyapunov-type inequalities for certain higher order differential equations. J. Math. Anal. Appl. 195, 527-536 (1995)

15. He, X, Tang, XH: Lyapunov-type inequalities for even order differential equations. Commun. Pure Appl. Anal. 11, 465-473 (2012)

16. Youmei, Z, Qingling, Z, Tamaki, T, Min, C: Admissibility for positive continuous-time descriptor systems. Int. J. Syst. Sci. 44, 2158-2165 (2013)

17. Agarwal, RP, Özbekler, A: Lyapunov type inequalities for even order differential equations with mixed nonlinearities. J. Inequal. Appl. 2015, 142 (2015)

18. Cañada, A, Montero, JA, Villegas, S: Lyapunov-type inequalities and applications to PDE. In: Elliptic and Parabolic Problems. Progr. Nonlinear Differential Equations Appl., vol. 63, pp. 103-110. Birkhäuser, Basel (2005)

19. Cañada, A, Montero, JA, Villegas, S: Lyapunov inequalities for partial differential equations. J. Funct. Anal. 237, 176-193 (2006)

20. Hashizuma, M, Takahashi, F: Lyapunov inequality for an elliptic problem with the robin boundary condition. Preprint

21. Timoshin, SA: Lyapunov inequality for elliptic equations involving limiting nonlinearities. Proc. Jpn. Acad. Ser. A. 86, 139-142 (2010)

22. Anastassiou, GA: Multivariate Lyapunov inequalities. Appl. Math. Lett. 24, 2167-2171 (2011)

23. de Nápoli, PL, Pinasco, JP: Lyapunov-type inequalities for partial differential equations. arXiv:1304.6988 [math.AP]

24. de Nápoli, PL, Pinasco, JP: Estimates for eigenvalues of quasilinear elliptic systems. J. Differ. Equ. 227, 102-115 (2006)

\section{Submit your manuscript to a SpringerOpen ${ }^{\circ}$ journal and benefit from:}

- Convenient online submission

Rigorous peer review

- Immediate publication on acceptance

- Open access: articles freely available online

- High visibility within the field

- Retaining the copyright to your article 